\title{
Brillouin Rectangular Optical Filter With Improved Selectivity and Noise Performance
}

\author{
Wei Wei, Lilin Yi, Yves Jaouën, Michel Morvan, and Weisheng Hu
}

\begin{abstract}
We demonstrate a high selectivity rectangular optical filter based on stimulated Brillouin scattering (SBS) effect in fiber with bandwidth from 1 to 3 GHz. By using pump-splitting dual-stage configuration, the filter selectivity has been improved by more than $16 \mathrm{~dB}$ and exceeded $40 \mathrm{~dB}$ within 2-GHz bandwidth. The filter shape is well controlled by using precise digital feedback compensation and nonlinearity management. We also analyze the noise performance of both the single-stage and dual-stage filters by evaluating the signal-tonoise ratio of the amplified signal using coherent detection. The results show that the dual-stage approach performs better than the single-stage architecture in achieving higher selectivity and improving noise performance. The inherent tuning flexibility with improved performance makes the proposed SBS filter one of the best candidates for microwave photonic applications.
\end{abstract}

Index Terms-Rectangular optical filter, microwave photonic filter, high selectivity, stimulated Brillouin scattering.

\section{INTRODUCTION}

W ITH the prosperity of the microwave photonics where radio frequency systems can take advantage of optical techniques, microwave photonic filter (MPF) has become an important component which can bring a lot of benefits such as low loss, immunity to electromagnetic interference (EMI), tunability, and reconfigurability [1]. MPFs have been usually realized by different kinds of photonic delay lines [2], [3]. Moreover, narrowband optical filters can also be used to implement MPFs such as micro-ring resonators [4] and stimulated Brillouin scattering (SBS) [5]. Among all the MPFs, single passband rectangular response and high selectivity are always highly desired as they allow to keep highest signal fidelity and filter out most of unwanted noise.

Recently, we demonstrated a rectangular optical filter based on SBS effect in fiber [6]. The Brillouin pump was generated using a multi-frequency electrical comb. We had successfully

Manuscript received December 8, 2014; revised April 14, 2015; accepted May 6, 2015. Date of publication May 12, 2015; date of current version July 7, 2015. This work was supported in part by the National Basic Research Program of China under Grant 2012CB315602, in part by the Program of Excellent Ph.D. in China under Grant 201155, in part by the Futur et Ruptures Program through the Institut Télécom of France, and in part by the National Natural Science Foundation of China under Grant 61132004 and Grant 61322507.

W. Wei, L. Yi, and W. Hu are with the State Key Laboratory of Advanced Optical Communication Systems and Networks, Shanghai Jiao Tong University, Shanghai 200240, China (e-mail: weiweimmc@sjtu.edu.cn; lilinyi@sjtu.edu.cn; wshu@sjtu.edu.cn).

Y. Jaouën is with Institut Mines-Télécom, Télécom ParisTech, Paris 75634, France (e-mail: yves.jaouen@telecom-paristech.fr).

M. Morvan is with Institut Mines-Télécom, Télécom Bretagne, Technopôle Brest-Iroise, Brest 29238, France (e-mail: michel.morvan@ telecom-bretagne.eu).

Color versions of one or more of the figures in this letter are available online at http://ieeexplore.ieee.org.

Digital Object Identifier 10.1109/LPT.2015.2432115 achieved rectangular filter shape with precise digital feedback compensation and nonlinearity management. The filter bandwidth could be tuned from $50 \mathrm{MHz}$ to $4 \mathrm{GHz}$. Compared with the sweeping pump based method in [5], our approach could control the filter shape more precisely by controlling the amplitude and initial phase of each electrical comb line. But the selectivity of the filter was not very high due to both the competition between SBS and Stimulated Raman effect (SRS) and four wave mixing (FWM) induced out-of-band gain. These two issues were particularly severe in large bandwidth cases.

In this letter we propose a pump-splitting dual-stage scheme to increase the filter selectivity. We compare the single- and dual-stage configurations for the filter bandwidth of 1,2 , and $3 \mathrm{GHz}$. The selectivity has been improved by as high as $\sim 16 \mathrm{~dB}$ and exceeded $40 \mathrm{~dB}$ within $2-\mathrm{GHz}$ bandwidth without any distortion of shape. It makes the filter more practical and suitable for more applications. We also analyze the noise performance of both single- and dual-stage filters by amplifying a single-tone signal and measuring the signal to noise ratio (SNR). The results show that when the SBS gain is large, noise performance of the dual-stage approach is better than the single-stage case. Thus the proposed filter has all the desired characteristics that an MPF needs: high selectivity, rectangular shape, flexibility of bandwidth and frequency tuning.

\section{PRINCIPLE}

The proposed SBS filter includes two key points: first the pump generation method and second the filter shape control [6]. Since it is extremely difficult to directly control the optical spectrum shape, we first use an arbitrary waveform generator (AWG) to generate an electrical comb which then modulates a CW light acting as the pump wave. This pump generation method ensures the precise control of the SBS pump spectrum. Then a feedback compensation algorithm is well designed to digitally control the amplitude of each electrical line in the comb in order to control the shape of the targeted SBS filter response. Meanwhile, unequal interval comb is used to mitigate the in-band FWM and improve the passband flatness. We call it nonlinearity management. By all this means, a rectangular optical filter has been achieved.

However, the filter selectivity cannot be increased by simply increasing pump power due to two reasons. The first reason is the competition between SBS and SRS effect. The Brillouin and Raman gains $\mathrm{G}_{\mathrm{B} / \mathrm{R}}$ both can be written as (1) [7]:

$$
G_{B / R(o n / o f f)}=\exp \left(\frac{g_{B / R} P_{o} L_{e f f}}{A_{e f f}}\right)
$$

where $\mathrm{g}_{\mathrm{B} / \mathrm{R} \text { (on/off) }}$ is the on-off Brillouin/Raman gain coefficient, $\mathrm{P}_{0}$ is the pump power, $\mathrm{L}_{\text {eff }}$ and $\mathrm{A}_{\text {eff }}$ are the effective 

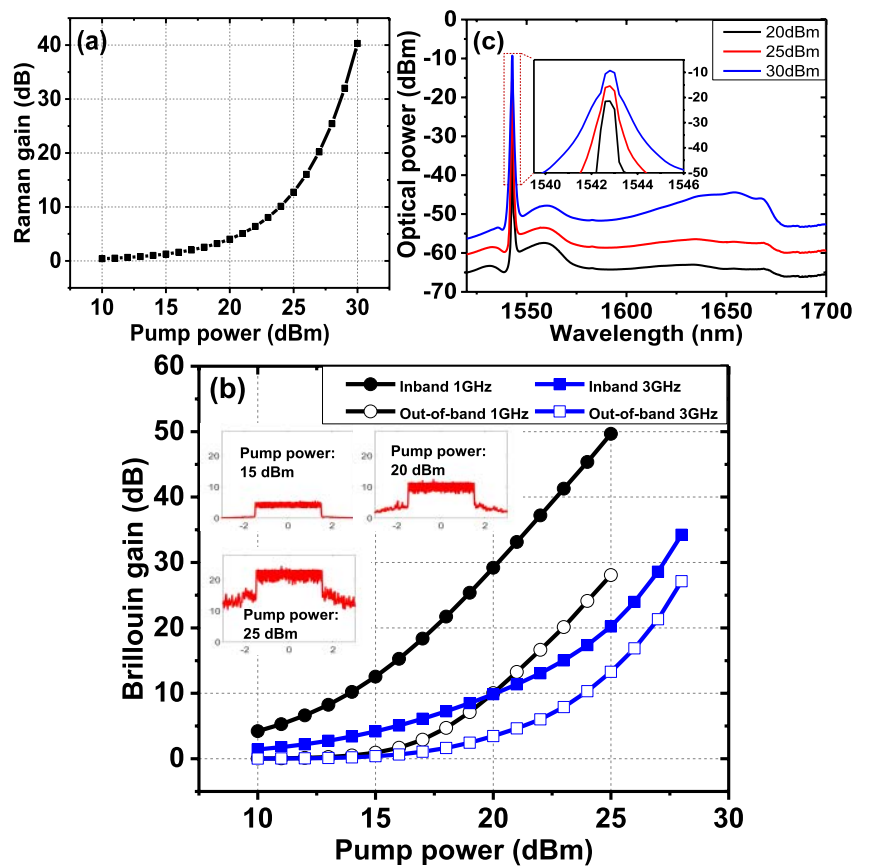

Fig. 1. Two limitations on improving filter selectivity. (a) Increasing Raman effect with pump power. (b) Increasing out-of-band gain induced by FWM at the same increasing pace as the inband gain with the increase of the pump power. Insets: Brillouin gains with different pump power after feedback process. (c) The output spectra of SBS amplifier without input signal.

fiber length and mode area respectively. When the Brillouin pump power is higher than several hundreds of $\mathrm{mW}$, it starts acting as Raman pump at the same time which results in SBS pump power consumption. Figure 1(a) shows the Raman gain obtained using formula (1), which increases dramatically once the pump power exceeds $\sim 23 \mathrm{dBm}$. The second reason for filter selectivity limitation is FWM effect induced by multi-frequency comb. It generates new components out of the filter passband and cannot be completely mitigated by the nonlinearity management method. When the pump power increases, FWM effect is getting much more severe especially in large bandwidth cases. The out-of-band gain will consume considerable amount of pump power and disrupt the rectangular shape generation. Thus when the pump power continues to increase, most of the power is wasteful and brings about hardly no benefit.

We have conducted numerical simulations to further prove the pump power limitation induced by FWM. For simplicity, we only focus on the pump wave variation during the fiber propagation in a $25-\mathrm{km}$ long single mode fiber (SMF). A scalar model of fiber propagation based on the Split-Step Fourier resolution method has been considered [7]. The fiber parameters are $0.2 \mathrm{~dB} / \mathrm{km}$ for fiber loss, $17 \mathrm{ps} / \mathrm{nm} / \mathrm{km}$ for chromatic dispersion and $1.3 \mathrm{~W}^{-1} \mathrm{Km}^{-1}$ for the non-linear parameter respectively. The total SBS gain is obtained by accumulating the SBS gain of each short fiber slice which is calculated using formula (1). Fig. 1(b) illustrates the relation between the pump power and the SBS gain. We use the gain at $100 \mathrm{MHz}$ away from the filter edges to represent the out-of-band gain level. For both $1-\mathrm{GHz}$ and $3-\mathrm{GHz}$ bandwidth cases, the out-ofband gain increases at the same pace as the inband gain. The insets of Fig. 1(b) demonstrate the gain shape of 3-GHz filters with different pump power after feedback process. Even though the gain increases from 10 to $20 \mathrm{~dB}$ when the pump power increases from 20 to $25 \mathrm{dBm}$. The out-of-band gain also increases by $10 \mathrm{~dB}$, which makes the two filters almost the same shape. Thus the SBS filter selectivity cannot be increased at all by increasing the pump power. Note that the real Brillouin gain at large pump power cases may be smaller than the simulation results considering the pump depletion. Figure 1(c) shows the measured spectra of 3-GHz SBS amplifier output when the input signal is turned off. The peak around $1543 \mathrm{~nm}$ corresponds to the Rayleigh backscattering of the pump. When the pump power is increased to $30 \mathrm{dBm}$, a typical silica-based Raman spectrum is clearly observed at $\sim 100 \mathrm{~nm}$ away from the pump wave. Meanwhile, in the inset, the pump spectra are broadened with the increase of the pump power due to the out-of-band FWM effect. Therefore the SBS gain at large bandwidth cannot be effectively increased by simply increasing the pump power.

In order to increase the filter selectivity, we propose a pumpsplitting dual-stage scheme. Instead of using a single pump with high power, we split it into two stages and amplify the signal twice successively. The split pump can still be adjusted with the same feedback control method. Note that the filter selectivity is only related to the On-Off gain and has nothing to do with the transmission loss, extra attenuation can be induced between the 2 stages to prevent the signal saturation in the second stage. In this case, the pump power of each stage is not too high to stimulate high Raman gain nor to induce too much out-of-band FWM components. Moreover, the decrease in pump power for each stage will decrease the induced noise from the spontaneous Brillouin emission [8]. Thus the filter selectivity can be increased dramatically and better noise performance can be achieved at the same time.

The fiber we use is SMF. Compared with fibers with multiple Brillouin peaks such as Dispersion compensation fiber (DCF), SMF has only one obvious Brillouin peak, which will improve the filter passband flatness. Although SMF has larger effective mode area which makes it less efficient to generate SBS gain than DCF, the FWM effect and Raman gain are decreased in the same way. As to the fiber length, less pump power is needed to obtain the same gain in longer fiber, but on the contrary in this case the FWM will be enhanced in the meantime. Thus there is an optimal fiber length considering the trade-off between the Brillouin gain and FWM. In our experiment, two identical fibers (i.e. same SBS characteristics) of same length are preferable. Due to lab constraints, we used two identical 25-km SMF28.

\section{RECTANGUlaR FILTER With IMPROVED SELECTIVITY}

The experimental setup is shown in Fig. 2. A distributed feedback (DFB) laser operating at $\sim 1543 \mathrm{~nm}$ is split into two branches for the generation of pump and probe signal in the upper and lower branch respectively. In the upper branch, an AWG is used to generate electrical spectral lines with random frequency interval within $\pm 1-\mathrm{MHz}$ deviation from the natural SBS bandwidth of $20 \mathrm{MHz}$, (i.e. $19 \mathrm{MHz}, 20 \mathrm{MHz}$ and $21 \mathrm{MHz}$ ). Then it modulates the $\mathrm{CW}$ light to generate the optical carrier-suppressed single-sideband (OCS-SSB) 


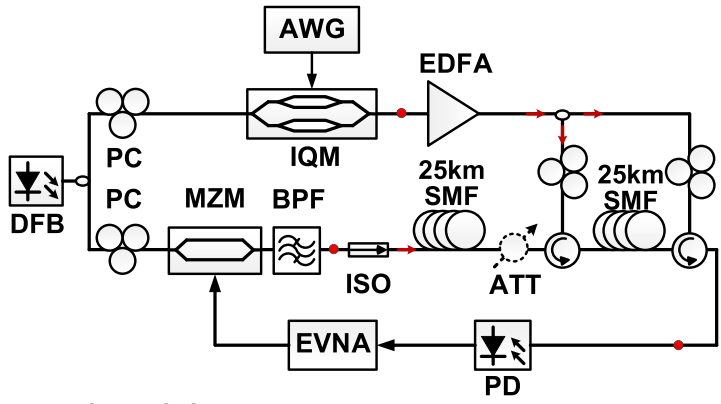

(i)

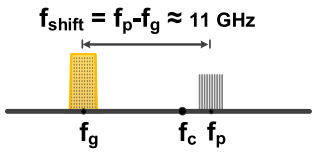

(iii)

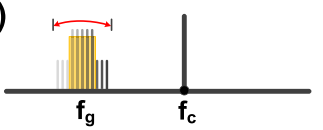

(ii)

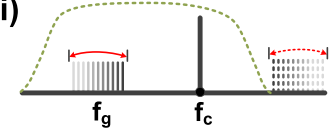

(iv)

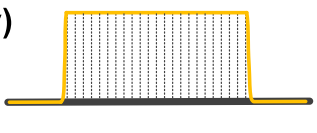

Fig. 2. Experimental setup. Inset (i) single sideband pump $f_{p}, S B S$ gain around $f_{g}$ and DFB laser frequency $f_{c}$, (ii) sweeping probe signal, (iii) sweeping probe signal amplified by the SBS gain, (iv) measured gain spectrum.

SBS pump lines utilizing an I\&Q modulator (IQM). After boosted by a high power erbium-doped fiber amplifier (EDFA), the OCS-SSB signal is then split into two parts equally and send into two identical 25-km long SMFs, which are under the same strain and temperature conditions to ensure the same Brillouin characteristics. In each stage, a polarization controller (PC) is used to maintain the SBS gain at the maximum value. For the single-stage case, we only use the first $25-\mathrm{km}$ SMF and launch all the pump power in it. The SBS gain is $\sim 11-\mathrm{GHz}$ away from the pump as shown in Fig. 2(i). In the lower branch, a sweeping signal covering the whole SBS gain region from an electrical vector network analyzer (EVNA) modulates the CW light utilizing a Mach-Zehnder modulator (MZM) to generate the probe signal. After suppression of the left sideband by an optical bandpass filter (BPF) as shown in Fig. 2(ii), the probe light propagates in the two fibers successively and is amplified once it has swept within the SBS gain region as shown in Fig. 2(iii). An optical attenuator (ATT) between the two stages can prevent signal saturation in the second stage therefore effectively increase the filter selectivity. The probe signal is then detected by a photodiode (PD) and sent into the EVNA. The amplitude response sketched in Fig. 2(iv) is measured by the EVNA and the SBS gain spectrum can be obtained by comparing the results between the SBS pump on and off.

The amplitude and phase responses of both single- and dual-stage filters are shown in Fig. 3. The pump power is set to 21,24 and $27 \mathrm{dBm}$ for filter bandwidth of 1,2 and $3 \mathrm{GHz}$ respectively. As shown in Fig. 3(a), for all bandwidth cases, the filter gain has been improved obviously with the same total pump power while the passband flatness remains at the same level. The larger the bandwidth is, the larger the increase is. For the 3-GHz filter, the maximum gain of single-stage filter is limited to $\sim 18.5 \mathrm{~dB}$ due to SRS and the severe out-of-band FWM components. By using the dual-stage scheme, the gain has been increased to $\sim 32 \mathrm{~dB}$. The high selectivity makes the filter shape more like rectangular than flat-top trapezoid. For the 1-GHz dual-stage filter, by using dual-stage pump not only
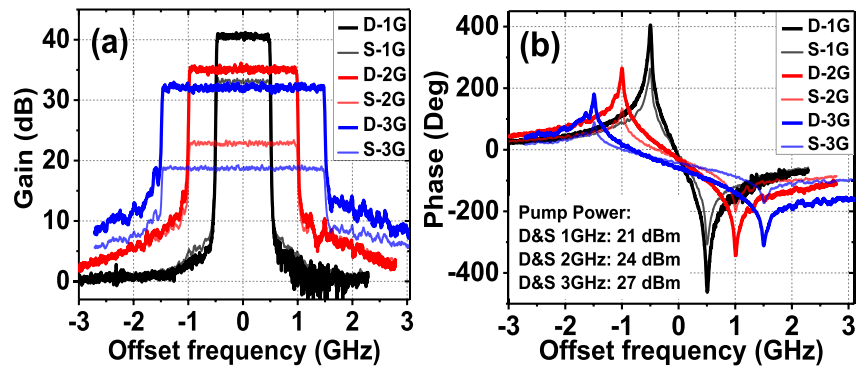

Fig. 3. Filter comparison between single- and dual-stage approaches. (a) The amplitude and (b) phase responses of the 2 types of filter. D: dual-stage; S: single-stage.

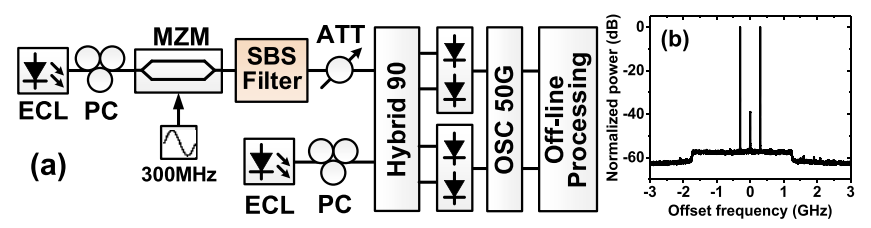

Fig. 4. (a) Noise measurement setup and (b) the electrical spectrum of the amplified signal by a $3-\mathrm{GHz}$ SBS filter.

is the gain increased, but also the out-of-band FWM effect is slightly mitigated. Note that the large noise at the right stop-band of the 1-GHz dual-stage filter is the beating noise of the probe signal and the Rayleigh backscattering of the pump, which is only due to the measurement method. As to the phase response shown in Fig. 3(b), all the curves are very smooth due to the small passband ripple, and higher gain of the dualstage filter leads to larger phase variation compared with the single-stage case. The results have clearly proved the validity of the dual-stage scheme to increase the filter selectivity.

\section{NOISE Performance}

When the pump is split into two parts, the decrease in pump power for each stage will decrease the induced noise to some degree. In order to verify this, we analyze the noise performance of the proposed SBS filter with the experimental setup shown in Fig. 4(a). A 300-MHz single-frequency signal modulates the $\mathrm{CW}$ light from an external cavity laser (ECL). After passing through the active SBS filter and an optical attenuator to maintain same input signal power level, the signal is detected by a coherent receiver. Figure 4(b) shows the spectrum of the SBS amplified signal which is calculated from the temporal waveform obtained by a real-time oscilloscope from a coherent receiver since we do not have a high-resolution optical spectrum analyzer. The symmetric frequency line is due to the carrier-suppressed double-sideband modulation by the Mach-Zehnder modulator. We do not use the CW light as the signal since the DC noise from the oscilloscope itself is significant. The Brillouin noise pedestal is off-center due to the slight frequency detuning between pump and signal waves.

Firstly, the relation between pump power and SBS gain has been measured as shown in Fig. 5. With the same total pump power, the dual-stage scheme brings about much higher gain. While the gain increase slows down in the single-stage case, the increase is still linear for the dual-stage scheme. If a $10 \mathrm{~dB}$ attenuation is introduced between the two stages, the maximum On-Off gain is increased by more than $16 \mathrm{~dB}$ when the filter bandwidth is 1 or $2 \mathrm{GHz}$. Note that the SBS gain also depends on the input signal power where lower input 


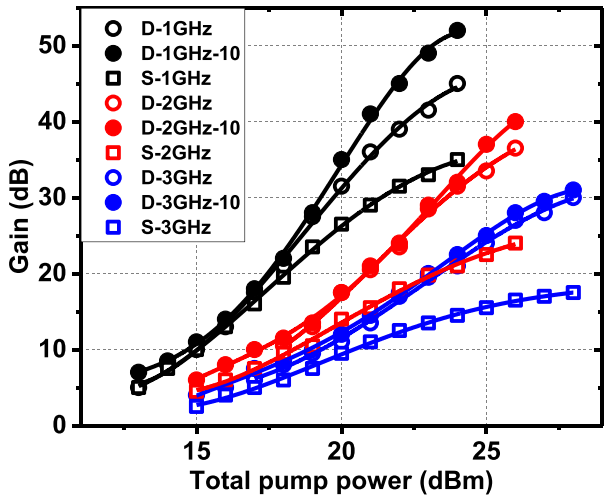

Fig. 5. The relation between pump power and SBS gain. D: dual-stage; S: single-stage. " -10 " means $10 \mathrm{~dB}$ optical attenuation between two stages.
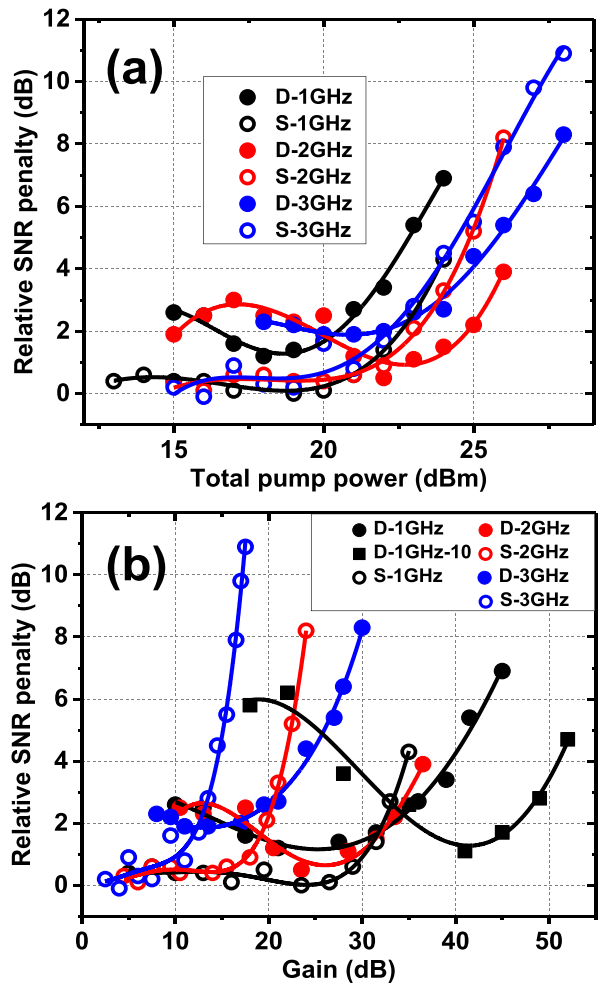

Fig. 6. Noise comparison between the single- and dual-stage filters. (a) The relation between SNR and pump power, (b) the relation between SNR and SBS gain. D: dual-stage; S: single-stage. "-10" means $10 \mathrm{~dB}$ optical attenuation between two stages.

power leads to larger gain. In the above measurements, we fix the input signal power level at $-20 \mathrm{dBm}$.

Then the SNR is carefully measured. We use the amplified signal peak power and the average noise power in the SBS gain region to calculate the SNR. We define the SNR penalty at $1-\mathrm{GHz}$ bandwidth with $19-\mathrm{dBm}$ pump power as reference since the SNR penalty under this condition is not obvious compared with the back-to-back case. The relation between the pump power and the SNR is shown in Fig. 6(a). We can conclude that the general trend of SNR penalty increases with the increase of the pump power and the pump power level is the main factor to induce noise. For the single-stage filter, the turning point is around $20 \mathrm{dBm}$, and the three curves show strong consistency. As to the dual-stage filter, the turning points generally shift 2 to $3 \mathrm{~dB}$ higher in both the $2-\mathrm{GHz}$ and $3-\mathrm{GHz}$ cases while in $1-\mathrm{GHz}$ case, the turning point remains the same. So if we can improve the pump efficiency to reduce the required pump power for the same gain value, the SNR performance can be improved. From this regard, the pump-splitting dual-stage configuration is preferred. Figure 6(b) demonstrates the SNR variation with different SBS gains. We can observe steep rise in SNR penalty with the increase of SBS gain after a certain turning point for both single- and dual-stage cases. For the small gain cases, the dual-stage induced SNR penalty is only 1 to $2 \mathrm{~dB}$. Once the gain reaches 32,19 , and $13 \mathrm{~dB}$ for the bandwidth of 1,2 , and $3 \mathrm{GHz}$ respectively, the noise performance of the dual-stage scheme are always better than the single-stage method. If $10-\mathrm{dB}$ attenuation between the two stages is used, the SNR will degrade when the gain is mall, but when the gain reaches $34.5 \mathrm{~dB}$, it induces smallest penalty and becomes the best choice. Note that for a SBS-based MPF, high selectivity with low noise is preferred, thus the pump-splitting dual-stage approach is a simple but effective method to reach this goal.

\section{CONCLUSION}

We have demonstrated a high selectivity narrowband rectangular optical filter based on stimulated Brillouin scattering. By splitting the pump into two stages and amplifying the signal twice successively, the filter selectivity has been improved dramatically, which makes the rectangular shape more close to the ideal one. We have also compared the noise performance between the single- and dual-stage scheme by amplifying a single frequency signal and obtaining the SNR with coherent detection. The dual-stage approach of SBS amplification shows its evident superiority in high gain conditions. By increasing the selectivity to higher than $40 \mathrm{~dB}$ within 2-GHz bandwidth with minor noise degradation, the proposed filter can be practically used in the field of microwave photonics and also for optical signal filtering and processing.

\section{ACKNOWLEDGMENT}

The authors thank Keopsys for providing a $30 \mathrm{dBm} \mathrm{Er} / \mathrm{Yb}$ high power amplifier.

\section{REFERENCES}

[1] J. Capmany, B. Ortega, and D. Pastor, "A tutorial on microwave photonic filters," J. Lightw. Technol., vol. 24, no. 1, pp. 201-229, Jan. 2006.

[2] Y. Dai and J. Yao, "Nonuniformly-spaced photonic microwave delay-line filter," Opt. Exp., vol. 16, no. 7, pp. 4713-4718, Mar. 2008.

[3] V. R. Supradeepa et al., "Comb-based radiofrequency photonic filters with rapid tunability and high selectivity," Nature Photon., vol. 6, no. 3, pp. 186-194, Mar. 2012.

[4] J. Palaci, G. E. Villanueva, J. V. Galán, J. Marti, and B. Vidal, "Single bandpass photonic microwave filter based on a notch ring resonator," IEEE Photon. Technol. Lett., vol. 22, no. 17, pp. 1276-1278, Sep. 1, 2010.

[5] Y. Stern et al., "Tunable sharp and highly selective microwave-photonic band-pass filters based on stimulated Brillouin scattering," Photon. Res., vol. 2, no. 4, pp. B18-B25, Aug. 2014.

[6] W. Wei, L. Yi, Y. Jaouën, and W. Hu, "Bandwidth-tunable narrowband rectangular optical filter based on stimulated Brillouin scattering in optical fiber," Opt. Exp., vol. 22, no. 19, pp. 23249-23260, Sep. 2014.

[7] G. P. Agrawal, Nonlinear Fiber Optics. Waltham, MA, USA: Academic, 2013, pp. 297-300 and 358-359.

[8] N. A. Olsson and J. P. van der Ziel, "Characteristics of a semiconductor laser pumped Brillouin amplifier with electronically controlled bandwidth," J. Lightw. Technol., vol. 5, no. 1, pp. 147-153, Jan. 1987. 